\title{
Cryptogenic Hepatitis: A Case Study in Autoantibody Negative Autoimmune Hepatitis
}

\author{
VINCENT GACAD, KATHY WALLER
}

\begin{abstract}
A 76 year old white-female initially presented with signs and symptoms of acute hepatitis. While liver function tests were elevated, viral hepatitis and autoimmune hepatitis panels were negative. The patient was subsequently discharged on the $4^{\text {th }}$ day of hospitalization when her condition appeared to stabilize. Four weeks later she was readmitted to the hospital, this time with signs and symptoms of acute liver failure with ascites, confusion, and jaundice. A liver biopsy was performed with pathology suggestive of an autoimmune etiology despite the negative autoimmune antibody panel. She was diagnosed with cryptogenic autoimmune hepatitis. The patient was placed on intravenous steroids and later oral prednisone that resulted in the resolution of the liver failure and disappearance of the encephalopathy along with a downward trend of the liver enzymes, bilirubin and the prothrombin time. One year later, the liver enzymes had normalized and the autoimmune disease remained in remission with maintenance medications consisting of low dose prednisone and azathioprine.
\end{abstract}

ABBREVIATIONS: AAA - anti-actin antibody; AIH Autoimmune Hepatitis; ALC-1 - anti-liver cytosol-1; ALT-alanine transaminase; ALP- alkaline phosphatase; AMA - antimitochondrial antibody; ANA - antinuclear antibody; ASMA - anti-smooth muscle antibody; AST aspartate transferase; CBC - complete blood count; COPD - Chronic Obstructive Pulmonary Disease; $\mathrm{H} \& \mathrm{E}$ - hematoxylin and eosin; IIF - indirect immunofluorescence; LKM-1 - liver/kidney microsomal antibody Type-1; pANCA - perinuclear antineutrophil cytoplasmic antibody; PT - prothrombin time.

INDEX TERMS: Acute Hepatitis, Autoimmune Hepatitis, Cryptogenic Hepatitis.

Clin Lab Sci 2011;24(4):196
Vincent Gacad, The Ohio State University, Columbus, $\mathrm{OH}$

Kathy V. Waller, Ph.D., MLS, The Ohio State University, Columbus, $\mathrm{OH}$

Address for Correspondence: Vincent Gacad, The Ohio State University, (440) 832-1043, gacad.1@buckeyemail. osu.edu

\section{CASE HISTORY}

A 76 year old white female was admitted to the hospital after two weeks of increasing nausea and anorexia, without fever or chills. The patient is a homemaker with known COPD and no cardiac problems. She denied any medication intake, including over-thecounter drugs (specifically Tylenol", nonsteroidal antiinflammatory drugs (NSAIDs), or aspirin). Two days prior to admittance, the patient noticed yellow discoloration of her eyes and tea colored urine. She had no prior history of alcohol use and smoked half a pack of cigarettes per day for 50 years. There was neither family history of liver disease nor any known autoimmune disease. A review of her signs and systems revealed that the patient has lost 20 pounds over the past two months; she had no chest pain, minimal pruritus, no stool discoloration, no diarrhea nor constipation and no history of rectal bleeding.

Upon admission, the patient presented with initial impression of acute hepatitis - "grossly icteric" eyes, jaundice, fatigue, and nausea. Her blood analyses showed elevated levels of bilirubin, AST, ALT, ALP and PT (Table 1). The total serum protein was normal, with a mildly elevated globulin level and a decreased albumin. Her CBC showed a normal leukocyte count, hemoglobin, and platelet count. An acute viral hepatitis panel analysis was performed and the patient was negative for Hepatitis A, B, and C. A drug toxicity 


\section{CLINICAL PRACTICE}

Table 1. Significant Laboratory Results for this Case

\begin{tabular}{|c|c|c|c|c|c|c|c|c|}
\hline Analyte & Reference Range & Admit & Discharge & Outpt & Outpt & $\begin{array}{l}\text { Admit } \\
\mathrm{P}^{\mathrm{B}}, \mathrm{I}^{\mathrm{C}}\end{array}$ & $\begin{array}{c}\text { Discharge } \\
\mathrm{P}^{\mathrm{B}}, \mathrm{I}^{\mathrm{C}}\end{array}$ & $\begin{array}{l}\text { Outpt }{ }^{\mathrm{A}} \\
\mathrm{I}^{\mathrm{C}} \text { Only }\end{array}$ \\
\hline & & Day 1 & Day 4 & Day 29 & Day 33 & Day 36 & Day 50 & Day 377 \\
\hline Total Protein & $6.0-9.3 \mathrm{~g} / \mathrm{dL}$ & 6.5 & 6.8 & 6.6 & 6.6 & 6.6 & 5.3 & 8.0 \\
\hline Albumin & $3.5-5.7 \mathrm{~g} / \mathrm{dL}$ & 2.5 & 3.0 & 3.4 & 3.0 & 2.5 & 1.8 & 4.1 \\
\hline Globulin & $1.7-3.4 \mathrm{~g} / \mathrm{dL}$ & 4.0 & 3.8 & 3.2 & 3.6 & 4.1 & 3.5 & 3.9 \\
\hline Bilirubin, Total & $0.3-1 \mathrm{mg} / \mathrm{dL}$ & 11.1 & 9.4 & 9.4 & 20.4 & 24.0 & 5.7 & 1.4 \\
\hline AST & $13-39 \mathrm{U} / \mathrm{L}$ & 461 & 495 & 740 & 1090 & 2835 & 133 & 26 \\
\hline ALT & $7-52 \mathrm{U} / \mathrm{L}$ & 8112 & 538 & 662 & 913 & 2544 & 323 & 42 \\
\hline ALP & 34-104 U/L & 139 & 145 & 179 & 185 & 219 & 195 & 0.3 \\
\hline Prothrombin Time & $9.4-11.1 \mathrm{sec}$. & 13.0 & 12.5 & $\mathrm{n} / \mathrm{a}$ & $\mathrm{n} / \mathrm{a}$ & 22.7 & 16.5 & $\mathrm{n} / \mathrm{a}$ \\
\hline $\begin{array}{l}\text { AIH Panel } \\
\text { (ANA, AMA, } \\
\text { ASMA, LKM-1) }\end{array}$ & Negative & Negative & $\mathrm{n} / \mathrm{a}$ & $\mathrm{n} / \mathrm{a}$ & $\mathrm{n} / \mathrm{a}$ & Negative & $\mathrm{n} / \mathrm{a}$ & Negative \\
\hline
\end{tabular}

Outpt = Outpatient

$\mathrm{n} / \mathrm{a}=$ not available

${ }^{\text {A }}$ Outpatient follow-up approx. one year after initial diagnosis. The patient is routinely seen for follow-up appointments every 6 months.

${ }^{\text {B }}$ Treatment with Prednisone

${ }^{\mathrm{C}}$ Treatment with Imuran

panel was also negative. An autoimmune panel was completed and the patient was negative for ANA, ASMA, and LKM-1. Although the immediate etiology of acute hepatitis was not evident, she was discharged after four days of clearing symptoms along with the observation of a downward trend in ALT without significant elevation of the PT test. The patient was able to sustain nutrition and hydration by mouth and there were no signs of encephalopathy, indicating stability of her condition.

The patient was readmitted four weeks later with apparent signs and symptoms of acute liver failure including jaundice, encephalopathy, ascites, nausea and fatigue. Her physician also noted a state of confusion, secondary to hepatic encephalopathy. Her laboratory tests showed higher levels of AST, ALT, ALP and PT than from the initial discharge from the hospital. The viral panel and autoimmune panel were repeated, and all tests remained negative. An abdominal paracentesis procedure revealed an accumulation of ascites fluid caused by portal hypertension secondary to cirrhosis. Histologic features of portal, periportal and lobular inflammatory infiltrates were highly suggestive of autoimmune disease. There were increased eosinophils and plasma cells, together with ballooning hepatocyte degeneration, and portal/lobular inflammation - all signs of autoimmune hepatitis (Figure 1). The patient was started on a regimen of IV steroids and later placed on tapered oral prednisone. She was discharged after 14 days, with slowly declining liver panel numbers, bilirubin and prothrombin time and resolution of symptoms.

Since the patient had increased fluid retention while on a higher dose of steroids, she was started on Azathioprine $50 \mathrm{mg}$ daily in combination with the tapered Prednisone dose to a maintenance dose of 10 mg per day. Therapy resulted in a continued decline and eventual normalization of all liver enzymes, bilirubin and prothrombin time with no obvious side effects from neither Prednisone nor Azathioprine.

Since discharge, the patient had follow-up visits with her physician every six months. She has remained asymptomatic and the autoimmune hepatitis remains in 


\section{CLINICAL PRACTICE}

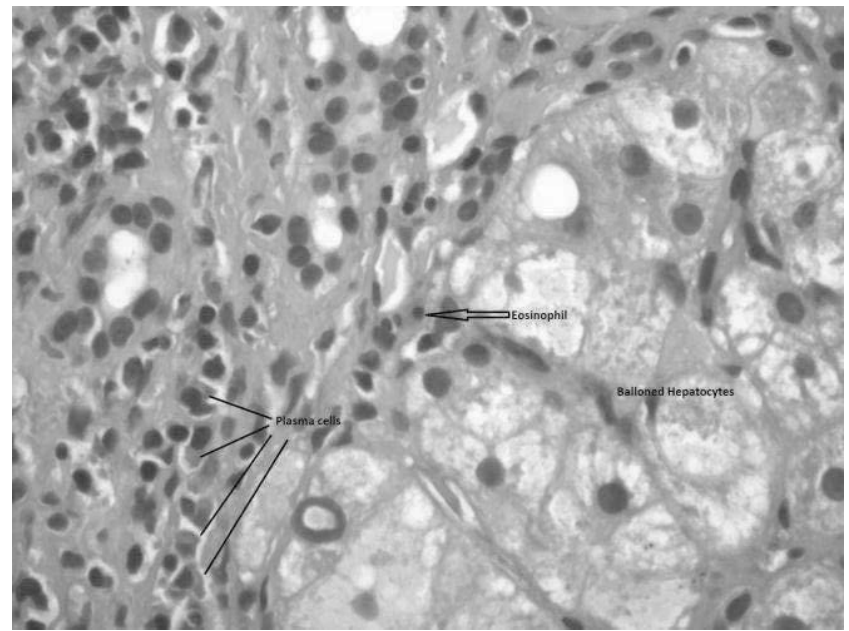

Figure 1. Ballooning hepatocytes with eosinophils and plasma cells (H\&E stain, 40x magnification)

remission with dual therapy of Azathioprine and low dose prednisone. Her encephalopathy and ascites have not recurred and an ultrasound of the liver showed no evidence of tumor. Unlike many $\mathrm{AIH}$ cases, her autoimmune titers were still negative when repeated.

\section{AUTOIMMUNE HEPATITIS (AIH)}

Autoimmune hepatitis is a liver inflammation of unknown etiology. Usually, AIH is a chronic, unresolving hepatitis that is commonly characterized by an onset of jaundice and elevation of aminotransferases, globulins, and autoimmune antibody titers. On liver biopsy and histology, AIH patients usually present with a lymphocytic inflammation and necrosis in the periportal zones. ${ }^{1}$ Although the progression mechanisms of AIH are not fully understood, it is believed that this condition results from an interaction among some predisposing factors (such as environment, age, sex, etc.), autoantigens, and immunoregulatory networks. Epidemiologic studies estimate the incidence of AIH to be 50-200 cases per million in Caucasian populations. ${ }^{2}$ The incidence of $\mathrm{AIH}$ is more prevalent in females (3.6:1). ${ }^{3}$ It has also been seen in younger populations, primarily; $40 \%$ of type 1 and $80 \%$ of type 2 AIH cases are diagnosed before age $18 .^{2}$

Recently, there have been studies that have examined the genetic factors that may contribute to an increased risk of developing AIH. It is believed that autoimmune hepatitis is genetically complex as it does not follow a Mendelian mode of inheritance and no single genetic locus has been identified. However, it is believed that the disease has an association with the Human
Leukocyte Antigen (HLA) genes. ${ }^{2}$ It is thought that these HLA genes are transcribed into proteins that ultimately influence presentation to the T-cell receptor at the peptide-binding groove. Slight genetic variations will affect amino acid sequence and therefore the peptide-binding groove. ${ }^{4}$

Recent studies reveal differences in gene coding between ethnic populations for AIH. In North America and Europe, susceptibility for AIH has been associated with HLA genes HLA-A1-B8, HLA-DRB1*0301, HLADRB1*0401(DR3 and DR4). ${ }^{2}$ Furthermore, a recent linkage disequilibriuim study has shown a preferential transmission of HLA-DRB ${ }^{*} 03$ (DR3), HLADRB*1301 (DR13), and HLA-DQB1*0201 to AIH patients compared to non-affected siblings. Other genetic studies propose HLA-DR13 to be a risk factor for AIH while in the absence of HLA-DR3 and HLADR4. ${ }^{2}$

In other populations, different HLA genes have been found to be risk factors for AIH. For example, HLADRB ${ }^{*} 0404$ is the predominant gene found in adult Mestizo-Mexican AIH patients. Argentina and Japan have an AIH population that expresses HLADRB $1 * 0405 .^{2}$ Although these genes are different, it is thought that they share a specific local genetic sequence, a "genetic motif." Among 94\% of Type 1 AIH patients, susceptibility genes have a specific six amino acid sequence of LLEQKR or LLEQRR at positions 67-72 on the DRB1 protein. ${ }^{2}$

\section{CLINICAL FEATURES}

Signs and Symptoms

Clinically, patients often present with nondiscriminatory symptoms, most notably fatigue. Jaundice can manifest in some patients, but only appears about $50 \%$ of the time. Other common symptoms include upper abdominal pain, anorexia, malaise, and arthralgia or myalgia, ascites, peripheral edema, hepatomegaly, splenomegaly, and encephalopathy. Rarely, this disease can manifest itself in an acute form. In the acute form, patients have a sudden onset of jaundice but do not feel unwell. This grave type of AIH must be diagnosed quickly, because a quick progression can cause acute liver failure. In approximately $15 \%$ of cases, patients will not show any outward symptoms; their diagnosis is usually incidental with an elevated aminotransferase level. ${ }^{5}$ 


\section{CLINICAL PRACTICE}

\section{Diagnosis}

The diagnosis of $\mathrm{AIH}$ requires the exclusion of conditions that are similar to AIH, while looking for characteristic features of the disease (usually found in biopsy and autoantibody tests). The histological appearance of AIH in biopsy tissue will be similar to that of chronic hepatitis of other etiologies (viral, druginduced, etc.). However, there are certain features that are characteristic (although not by themselves diagnostic) of AIH. Usually, an infiltrate of monocytes can be seen adjacent to the portal zones of the biopsy. It is believed that this interface hepatitis leads to lobular hepatitis. Many times, there is an abundance of eosinophils, but the eosinophil presence does not confirm or exclude diagnosis. As the disease progresses, a biopsy will usually show signs of extensive inflammation and fibrosis, leading to cirrhosis and eventually liver failure. ${ }^{3}$

One characteristic feature of $\mathrm{AIH}$ is its positive autoimmune antibody test results. Individuals with $\mathrm{AIH}$ exhibit multiple antibodies, the most common include antinuclear antibodies (ANA), anti-smooth muscle antibodies (ASMA), and liver/kidney microsome type 1 (anti-LKM1). There are two different serotypes of AIH (type 1 and 2), and each of these serotypes will have different positive autoantibody test results. Characteristic autoantibodies associated with type 1 AIH include ANA, ASMA, AAA, pANCA, and AMA, while those seen in type 2 AIH are LKM-1 and ALC. Antibodies against ANA and ASMA are widely considered the classical autoimmune markers of type 1 autoimmune hepatitis, and are present in $67 \%$ of all AIH cases. ${ }^{6}$

The nuclear reactivity of ANA is usually measured through indirect immunofluorescence (IIF) on Hep-2 cell lines. The ANA antigen and AIH antibodies will react against ribonucleoprotein, centromere, and ribonucleoprotein complexes, ${ }^{6}$ producing mostly a homogenous pattern in type $1 \mathrm{AIH} .{ }^{5}$ Antibodies against ASMA are positive in $87 \%$ of AIH patients, whether by itself, or in conjunction with ANA. ${ }^{6}$ These antibodies are specifically directed toward smooth muscle components including actin, tubulin, vimentin, desmin, and skeleton. ${ }^{6}$ A small percentage (3-4\%) of AIH cases will present with type-1 liver-kidney microsomal antibodies (anti-LKM1), without positive ANA or ASMA results. This type of AIH, known as type 2, has antibodies specifically directed against cytochrome isozyme P4502D6. Immunofluorescence of these antibodies show a characteristic staining pattern of renal tubules and granular cytoplasmic liver sections. ${ }^{5}$ Currently, ELISA kits are commercially available to test for all these autoantibodies. ${ }^{3}$

\section{Pathogenesis}

The human immune system has systems in place to differentiate self from non-self. Autoimmunity occurs when the body is unable to discriminate self from nonself-antigens, resulting in cross-reactivity. ${ }^{7} \mathrm{On}$ a cellular level, autoimmune liver damage is believed to be mediated by CD4+ $\mathrm{T}$ cells that recognize self-antigenic proteins. In order to trigger an immune response, the protein must connect to a human leukocyte antigen (HLA) class II molecule on the antigen-presenting cell and to an uncommitted CD4+ T-helper cell (Th0); the interaction between the two cells is stimulated by the interaction of CD28 on the Th0 cell and CD80 on the antigen-presenting cell. The Th0 cell then becomes activated, differentiating into one of two functional phenotypes (Th1 or Th2) according to the type of cytokines in the near environment and the nature of the presented antigen. These functional phenotypes have different roles in the immune system and the progression of AIH. Th1 cells activate macrophages by producing IL-2 and interferon $\gamma$, enhance the expression of HLA class I molecules, and induce expression of HLA class II molecules on hepatocytes. ${ }^{8}$ In total, Th1 cells make hepatocytes more vulnerable to CD8+ T-cell attack. Th2 cells produce IL-4, IL-10, and IL-13 interleukins that have been shown to stimulate autoantibody production by B-cells. ${ }^{8}$ In a normal individual, Th1 and Th2 cells antagonize each other, regulating the immune system; it is believed that an autoimmune attack occurs when these regulatory mechanisms fail. Studies have shown that HLA class II antigens have been found on AIH hepatocytes, while none are found on normal hepatocytes. Although numbers of these antigens tend to mimic the course of the disease, the mechanism by which they contribute to AIH is still unknown. ${ }^{7}$

\section{Treatment}

Untreated $\mathrm{AIH}$, with histological findings of bridging necrosis or multilobular necrosis, progresses to cirrhosis in $82 \%$ of patients, and has a mortality rate of $45 \%$ within three to five years of initial diagnsosis. ${ }^{3}$ 


\section{CLINICAL PRACTICE}

Treatments, however, seem to have a significant effect on patient prognosis. Studies have shown that the $20-$ year life expectancy for all treated patients is over $80 \%$, similar to the expectancy of age- and sex-matched normal subjects from the same geographical area. ${ }^{6}$

Immunosuppressive therapy with corticosteroids (most commonly prednisone) in combination with azathioprine $\left(\operatorname{Imuran}^{\mathrm{TM}}\right)$ is considered the gold standard for remission induction and maintenance. ${ }^{8}$ The observation of remission and maintenance of $\mathrm{AIH}$ is seen in the complete normalization of aminotransferase levels. Once normalization has occurred, it is recommended that the patient undergo a follow-up biopsy, because biochemical testing does not always fully correlate with the extent of liver injury. Although there is only minimal evidence for the length of time patients should be treated with corticosteroids, it has been proposed that patients be in stable remission for at least four years before therapy is withdrawn. ${ }^{9}$ Also, depending on case presentation, there are patients who would be recommended to stay on immunosuppressants for the rest of their life. ${ }^{3}$

Corticosteroid treatments have shown to induce clinical, laboratory, and histologic improvements in 60 $80 \%$ of patients with transplant-free survival at $90 \%$ after ten years. ${ }^{1}$ For patients who are considered corticosteroid resistant, recent studies have shown a regimen of cyclosporine to be an effective method of treatment. If the patient has severe cirrhosis from $\mathrm{AIH}$, liver transplant may be a viable option. ${ }^{6}$ Although the five-year patient and graft survival rate has reached $80 \%$, it is important to note that relapse is common when AIH therapy is stopped. After a patient has been in remission for 1-2 years, the chance of maintaining remission while withdrawing medication is only $30-$ $50 \% .^{1}$

\section{CRYPTOGENIC CHRONIC HEPATITIS}

Thirteen percent of nonviral-induced hepatitis patients will satisfy the signs and symptoms and serum protein criteria for autoimmune hepatitis, but will lack the antibodies characteristic to AIH (ANA, ASMA, LKM-1, etc.). ${ }^{10}$ These patients have been found to follow the same patient demographic as the classic AIH patient. ${ }^{11}$ Known as Cryptogenic (of unknown or indeterminate origin) Chronic Hepatitis, diagnosis is one of exclusion, by ruling out genetic liver diseases, alcoholic liver disease, drug toxicity, viral liver disease, and most importantly, cryptogenic liver disease (an advanced inactive non-discriminative cirrhosis in a liver transplant patient). ${ }^{5,6}$

In most cases of cryptogenic hepatitis, the patient will initially present with no detectable circulating autoantibodies; throughout the course of treatment with immunosuppressive therapy, these antibodies will appear. Although the cause of this phenomenon is unknown, it is believed that patients with severe liver damage will release massive amounts of tissue components, forming immune complexes, and ultimately blocking the detection of autoantibodies. ${ }^{5}$

\section{CLINICAL CORRELATION}

This patient's condition has been described as cryptogenic autoimmune hepatitis. It is usually characterized by having a clinical presentation of $\mathrm{AIH}$ without detectable autoimmune antibodies. However, the clinical presentation of this individual was not that of a typical AIH case. Autoimmune hepatitis is usually found in young women; she was older. She presented with an acute onset of a liver dyscrasia that moderated only somewhat after four days. In many "resolving" cases, the patient is usually discharged without the need for a biopsy. There was no reason to perform such an invasive procedure if her signs and laboratory values were subsiding. However, with negative autoantibody tests and no biopsy, there was little indication, at her first admission, that her condition was autoimmune.

When the patient was admitted the second time, she presented with acute liver failure. Like her first admission, this presentation was unusual since autoantibody testing and viral panel were negative. The next logical steps were to assess the liver damage and determine why the liver was failing. A biopsy showed the characteristic features of autoimmune hepatitis: portal and lobular inflammation, increased plasma cells and eosinophils, and ballooning hepatocytes with degeneration.

\section{CONCLUSION}

This is a case of a patient with Cryptogenic Autoimmune Hepatitis, an unusual form of AIH. However, she did not follow a normal cryptogenic presentation. The patient was treated with prednisone and azathioprine, normalizing her liver panel tests. Her autoantibody titers are retested every six months and 


\section{CLINICAL PRACTICE}

unlike the majority of cryptogenic AIH cases, still remain negative.

ACKNOWLEDGEMENTS: The authors wish to thank Dr. Reynaldo Gacad, Akron General Medical Center, Akron $\mathrm{OH}$ for sharing the patient's clinical data and review of the manuscript.

\section{REFERENCES}

1. Krok K, Munoz S. Management of autoimmune and cholestatic liver disorders. Clin Liver Dis 2009;13:295-316.

2. Beland K, Lapierre P, Alvarez F. Influence of genes, sex, age and environment on the onset of autoimmune hepatitis. World J Gastroenterol 2009;15:1025-34.

3. Manns MP, Czaja AJ, Gorham JD, Krawitt EL et al. Diagnosis and management of autoimmune hepatitis. Hepatology 2010;51:2193-213.

4 Strassburg C. Autoimmune hepatitis. Best Pract Res Clin Gastroenterol 2010;24:667-82.
5. McFarlane I. Autoimmune hepatitis: diagnostic criteria, subclassifications, and clinical features. Clin Liver Dis 2002;6:60521.

6. Czaja A, Freese D. Diagnosis and treatment of autoimmune hepatitis. Hepatology 2002;36:479-97.

7. Vergani D, Choudhuri K, Bogdanos D, Mieli-Veregani G. Pathogenesis of Autoimmune Hepatitis. Clin Liver Dis 2002;6:727-37.

8. Vergani D, Mieli-Vergani G. Autoimmune hepatitis and PSC connection. Clin Liver Dis 2008;12:187-202.

9. Teufel A, Galle P, Kanzler S. Update on autoimmune hepatitis. World J Gastroenterol 2009;15:1035-41. http://www. wjgnet.com/1007-9327/15/1035.pdf. Accessed 2011 Feb 24.

10. Czaja A. The variant forms of autoimmune hepatitis. Ann Intern Med 1996;125:588-98.

11. Czaja A, Bayraktar Y. Non-classical phenotypes of autoimmune hepatitis and advances in diagnosis and treatment. World J Gastroenterol 2009;15:2314-28.

The peer-reviewed Clinical Practice Section seeks to publish case studies, reports, and articles that are immediately useful, are of a practical nature, or contain information that could lead to improvement in the quality of the clinical laboratory's contribution to patient care, including brief reviews of books, computer programs, audiovisual materials, or other materials of interest to readers. Direct all inquiries to Perry Scanlan, PhD, MT(ASCP), Medical Technology, Austin Peay State University, Room D212, Sundquist Science Complex, Box 4668, Clarksville TN 37044.

Clinical Laboratory Science encourages readers to respond with thoughts, questions, or comments regarding these articles. Email responses to westminsterpublishers@comcast.net. In the subject line, please type the journal issue and lead author such as "CLIN LAB SCI 24(4) RE GACAD". Selected responses may appear in the Dialogue and Discussion section in a future issue. Responses may be edited for length and clarity. We look forward to hearing from you. 